\title{
Three-point Green Functions in the resonance region : LEC's *
}

\author{
J. Portolés ${ }^{\mathrm{a}}$

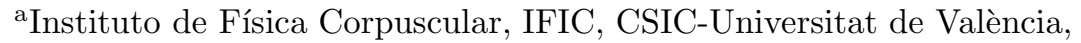 \\ Apt. Correus 22085, E-46071 València, Spain
}

Using the $1 / N_{C}$ expansion scheme and truncating the hadronic spectrum to the lowest-lying multiplets of resonances we develop a procedure to implement QCD information on the non-perturbative regime populated by resonances through the use of three-point Green Functions of QCD currents. The process implies to enforce on these functions the constraints provided by their asymptotic behaviour ruled by QCD using their OPE or associated form factors. As a result we are able to determine several couplings of the $\mathcal{O}\left(p^{6}\right)$ Chiral Perturbation Theory Lagrangian and, in consequence, to address phenomenological issues such as the $S U(3)$ breaking in $K_{\ell 3}$ decays.

\section{Introduction}

Quantum Chromodynamics (QCD) is, knowledgeably, the theory of strong interactions. However in spite of its early success in the description and understanding of the interaction between quarks and gluons, it soon became clear that, due to its asymptotic freedom property, the study of the low energy processes involving light-flavoured hadrons (typically $E \lesssim 2 \mathrm{GeV}$ ) would not be possible with a strong interaction theory written in terms of quarks and gluons as dynamical degrees of freedom. In the early eighties, and relying in a very fruitful heritage from the pre-QCD era, it was observed that the chiral symmetry of massless QCD could be used to construct a strong interaction field theory, intended to be dual to QCD, in terms of the lightest $S U(3)$ octet of pseudoscalar mesons in the role of pseudo-Goldstone bosons associated to the spontaneous breaking of that symmetry. This construction, known as Chiral Perturbation Theory $(\chi P T)$ 112, has been very much useful in the study of strong interaction effects at very low energy, where the theory has its domain, namely $E \ll M_{V}$ (being $M_{V}$ the mass of the $\rho(770)$, the lightest hadron not included in the theory). Its success has pervaded hadron physics

\footnotetext{
*Talk given at the High-Energy Physics International Conference on Quantum Chromodynamics, 2-9 July (2005), Montpellier (France); IFIC/05-40 report. To appear in the Proceedings.
}

in the last two decades, bringing to the main front the concept of effective field theory as a powerful tool to handle the non-perturbative regime of QCD. An effective field theory tries to embody the main features of the fundamental theory in order to handle the latter in a specific energy regime where it is, whether more inconvenient or just impossible, to apply it.

The $\chi$ PT Lagrangian has a perturbative structure guided by powers of the soft external momenta and light quark masses. Hence one can construct the theory up to a fixed order in the expansion, $\mathcal{O}\left(p^{n}\right)$, by adding to the previous terms the last order operators in $\mathcal{L}_{n}^{\chi P T}$, namely :

$\mathcal{L}_{\chi P T}=\mathcal{L}_{2}^{\chi P T}+\mathcal{L}_{4}^{\chi P T}+\mathcal{L}_{6}^{\chi P T}+\ldots$

The first term $\mathcal{L}_{2}^{\chi P T}$ embodies the spontaneous breaking of the chiral symmetry and depends only on two parameters : $F$, the decay constant of the pion, and $B_{0} F^{2}=-\langle 0|\bar{\psi} \psi| 0\rangle$, the vacuum expectation value of the light quarks; both of them in the chiral limit. Higher orders in the expansion bring in the information from short-distance contributions that have been integrated out, for instance resonance states. This information is incorporated into the low energy couplings (LECs) that weight the operators of the theory :

$\mathcal{L}_{4}^{\chi \mathrm{PT}}=\sum_{i=1}^{10} L_{i} \mathcal{O}_{i}^{(4)}$ 
$\mathcal{L}_{6}^{\chi \mathrm{PT}}=\sum_{i=1}^{90} C_{i} \mathcal{O}_{i}^{(6)}$

for three flavours. The explicit expressions for the operators can be read in Refs. 23]. The reference scale in the chiral expansion $\Lambda_{\chi} \sim M_{V}$ indicates that LECs in $\chi \mathrm{PT}$ should receive contributions from the energy regime at or above this scale. In Ref. 4 the contributions of the lightest multiplets of resonances to the $\mathcal{O}\left(p^{4}\right)$ LECs in $\mathcal{L}_{4}^{\chi P T}$ were computed and it was observed that they saturated the values extracted from phenomenological analyses. Hence it is sound to think that the most important contribution to the LECs is provided precisely by the energy region of the last integrated scale $\left(E \sim \Lambda_{\chi}\right)$.

\section{The Resonance Chiral Theory}

The phenomenology of hadron physics at low energies is starting to involve more precise predictions and, consequently, a good knowledge on the LECs becomes necessary. At $\mathcal{O}\left(p^{4}\right)$ a thorough study was carried out in Refs. 45] using a phenomenological Lagrangian that is intended to carry the information of QCD in the energy region of the hadronic resonances. The guiding principles that are implemented in their construction are the chiral symmetry, that describes the interactions of the Goldstone bosons, and the flavour symmetry that is collected in the representations of the resonance fields. This model-independent construction is called Resonance Chiral Theory $(\mathrm{R} \chi \mathrm{T})$. Schematically the structure of its operators is :

$\mathcal{O} \sim\left\langle R \ldots R \chi\left(p^{n}\right)\right\rangle$

where $R$ is short for a unitary representation of the resonance fields (the lightest multiplets of vector, axial-vector, scalar and pseudoscalar resonances), $\chi\left(p^{n}\right)$ is a chiral tensor of $\mathcal{O}\left(p^{n}\right)$ in the $\chi \mathrm{PT}$ counting, and the trace operates in the flavour space. As happens in $\chi \mathrm{PT}$ the symmetries do not give any information on the couplings of these operators that remain unknown from these settings. One of the most relevant properties of this construction is that, upon decoupling of the resonance fields, one recovers the structure of the $\chi \mathrm{PT}$ Lagrangian, hence allowing the writing of the $\chi \mathrm{PT}$ LECs in terms of the couplings of the $\mathrm{R} \chi \mathrm{T}$.

At $\mathcal{O}\left(p^{4}\right)$ one can see [4 that the only operators contributing to the LECs are those with one only resonance field and a chiral tensor $\mathcal{O}\left(p^{2}\right)$, $\left\langle R \chi\left(p^{2}\right)\right\rangle$. In the $U(3)$ limit only 6 operators with this structure appear. The construction of the $\mathrm{R} \chi \mathrm{T}$ to obtain the $\mathcal{O}\left(p^{6}\right)$ chiral LECs is much more involved and it requires, together with the previous ones, 70 operators with the structure $\left\langle R \chi\left(p^{4}\right)\right\rangle, 38$ operators $\left\langle R_{1} R_{2} \chi\left(p^{2}\right)\right\rangle$ and 7 operators with only resonance fields $\left\langle R_{1} R_{2} R_{3} \chi\left(p^{0}\right)\right\rangle$. These have been studied in Ref. [6].

We would like to emphasize that the use of $\mathrm{R} \chi \mathrm{T}$ helps to establish relations between LECs and also parameterizes them in terms of the new unknown couplings of the theory with resonance fields. However $\mathrm{R} \chi \mathrm{T}$, by itself, does not determine the LECs in $\chi \mathrm{PT}$. Therefore more information will be needed. On the other side $\mathrm{R} \chi \mathrm{T}$ plays an important role in the implementation of the large- $N_{C}$ ( $N_{C}$ is short for the number of colours) ideas as we will see in the following.

\section{LECs from Large- $N_{C}$ QCD}

The determination of the $\chi \mathrm{PT}$ LECs asks for a thorough knowledge of QCD in the energy region populated by light-flavoured resonances $\left(M_{V} \lesssim\right.$ $E \lesssim 2 \mathrm{GeV})$. This is a highly non-trivial issue that requires to master poorly known aspects such as bound and resonant states, duality and hadronization mechanisms, i.e. non-perturbative QCD. It has been known for long that the $S U\left(N_{C}\right)$ gauge theory in the $N_{C} \rightarrow \infty$ limit is more simple than the real theory while still resembling both qualitatively and quantitatively the $N_{C}=3$ case [7. The $1 / N_{C}$ expansion tells us that, at leading order, any Green Function of QCD currents (quark bilinears) can be given by a sum of all possible topologies of tree level diagrams allowed by an infinite spectrum of zero-width mesons. Consequently the final result for a Green Function in the $N_{C} \rightarrow \infty$ limit is an infinite sum of meromorphic functions (meson poles). Moreover this structure is further constrained as it has to satisfy Ward identities and other chiral symmetry restrictions for $E \ll M_{V}$ and it also may match the asymptotic behaviour ruled by perturbative QCD at $E \gg M_{V}$ [589. 
The interrelation between these different sources of information is the main goal of our project. Refs. 10 provide some seminal work on the link between the Large- $N_{C}$ expansion and resonance saturation.

A general property of the LECs in $\chi \mathrm{PT}$ is that they are coefficients of the Taylor expansion of some QCD Green Functions (once singularities associated with the contributions of low-momentum pseudoscalar intermediate states have been subtracted). The relevant correlation functions are, precisely, order parameters of the spontaneous breaking of chiral symmetry. Accordingly they do not receive contributions from perturbative QCD in the chiral limit and one expects LECs to be sensitive to the physics of the intermediate energy region $\left(\Lambda_{\chi}\right)$ [8].

Our proposal involves the use of the meromorphic approach to Green Functions, in terms of resonance or Goldstone states, in order to match its high-energy behaviour with the one provided by the Operator Product Expansion (OPE) at leading order in $\alpha_{S}$ and the momenta series. Hence we get information that we can translate into the LECs participating in the Green Function through its Taylor expansion in momenta. Several technical details follow :

- In order to construct the meromorphic procedure we include the lightest multiplets of resonances por $J^{P C}=0^{++}, 0^{-+}, 1^{--}, 1^{++}$ states, one for each channel. The cut in the spectrum means that we are not implementing properly the large- $N_{C}$ limit, though well known phenomenology suggests that higher masses contributions tend to be much suppressed.

- The determination of the meromorphic approach to the Green Function can be done in two different ways: on one side one can construct an ansatz that has the right properties; on the other a phenomenological Lagrangian, like $\mathrm{R} \chi \mathrm{T}$, can be employed to evaluate the Feynman diagrams that give the different topologies at tree level. Though both procedures are germane the second has the advantage of extracting information on the $\mathrm{R} \chi \mathrm{T}$ too, that later can be used in other set- tings.

- We try to match our construction of the Green Function with the leading order in its OPE and, in addition we can also enforce the asymptotic behaviour of related form factors (their vanishing at high transfer of momentum) as it results from the analysis of spectral functions [5]. It has been noticed [1] that, with a finite number of states, it may happen that inconsistencies between the conditions from form factors and OPE appear. When studying the $\langle V A P\rangle$ and the $\langle S P P\rangle$ Green functions ( $V$ is short for vector, $A$ for axial-vector, $S$ for scalar and $P$ for pseudoscalar currents) we have concluded that we were able to enforce both the asymptotic behaviour, for form factors involving stable final states, and the OPE constraints. However the corresponding restriction on form factors with resonances could not be compelled. We will have to deal with this feature that, in practice, turns out to be not too severe.

The application of this program in the study of three Green functions of QCD currents, namely $\langle V V P\rangle$ 12, $\langle V A P\rangle$ [13] and $\langle S P P\rangle$ 14] has been very rewarding.

\section{Example : $S U(3)$ breaking in $K_{\ell 3}$ decays}

$K_{\ell 3}$ decays offer one of the most accurate determinations of the $V_{u s}$ CKM element. The main uncertainty in extracting this matrix element comes from theoretical calculations of the vector form factor $f_{+}^{K^{0} \pi^{-}}(0)$ defined by :

$$
\begin{aligned}
\left\langle\pi^{-}(p)\left|\bar{s} \gamma_{\mu} u\right| K^{0}(q)\right\rangle= & f_{+}^{K^{0} \pi^{-}}(t)(q+p)_{\mu} \\
+ & f_{-}^{K^{0} \pi^{-}}(t)(q-p)_{\mu}
\end{aligned}
$$

with $t=(q-p)^{2}$. For $t=0$ deviations of $f_{+}^{K^{0} \pi^{-}}(0)$ from the unity (the octet symmetry limit) are of second order in $S U(3)$ breaking, hence

$f_{+}^{K^{0} \pi^{-}}(0)=1+f_{p^{4}}+f_{p^{6}}+\ldots$

The first correction is $\mathcal{O}\left(p^{4}\right)$ in $\chi \mathrm{PT}$ and it gives $f_{p^{4}}=-0.0227$ with virtually no uncertainty 
1516. At $\mathcal{O}\left(p^{6}\right)$ the situation is more complex as there appear contributions from two-loops with $\mathcal{L}_{2}^{\chi P T}$, one-loop with one vertex from the $\mathcal{L}_{4}^{\chi P T}$ Lagrangian, and tree-level diagrams with $\mathcal{L}_{4}^{\chi P T}$ and $\mathcal{L}_{6}^{\chi P T}$. These loops give [17] :

$f_{p^{6}}^{\text {loops }}\left(M_{\rho}\right)=0.0093 \pm 0.0005$.

Next, the tree level piece reads :

$$
\begin{aligned}
f_{p^{6}}^{\text {tree }}\left(M_{\rho}\right)= & 8 \frac{\left(M_{K}^{2}-M_{\pi}^{2}\right)^{2}}{F_{\pi}^{2}} \times \\
& {\left[\frac{\left(L_{5}^{r}\left(M_{\rho}\right)\right)^{2}}{F_{\pi}^{2}}-C_{12}^{r}\left(M_{\rho}\right)-C_{34}^{r}\left(M_{\rho}\right)\right], }
\end{aligned}
$$

that we can see involves the $\mathcal{O}\left(p^{4}\right) L_{5}$ and the $\mathcal{O}\left(p^{6}\right) C_{12}$ and $C_{34}$ LECs. In Ref. [14] we applied the procedure outlined in Section 3 for the study of the $\langle S P P\rangle$ Green function. We evaluated the contribution of one multiplet of scalar resonances of mass $M_{S}$ and one multiplet of pseudoscalar resonances of mass $M_{P}$, obtaining :

$$
\begin{aligned}
L_{5}^{S P} & =\frac{F^{2}}{4 M_{S}^{2}}, \quad C_{12}^{S P}=-\frac{F^{2}}{8 M_{S}^{4}}, \\
C_{34}^{S P} & =\frac{3 F^{2}}{16 M_{S}^{4}}+\frac{F^{2}}{16}\left(\frac{1}{M_{S}^{2}}-\frac{1}{M_{P}^{2}}\right)^{2},
\end{aligned}
$$

that provide a strong cancellation between the different contributions in $f_{p^{6}}^{\text {tree }}$. With the numerics discussed in that reference we get :

$$
\begin{aligned}
f_{p^{6}}^{\text {tree }}\left(M_{\rho}\right) & =-0.002 \pm 0.012 \\
f_{p^{6}} & =0.007 \pm 0.012 .
\end{aligned}
$$

The value of $f_{p^{6}}$ is in slight disagreement with that in Ref. [16]. We finally obtain :

$$
\begin{aligned}
f_{+}^{K^{0} \pi^{-}}(0) & =0.984 \pm 0.012 \quad \text { (10) } \\
\left|V_{u s}\right| & =0.2201 \pm 0.0027_{f_{+}(0)} \pm 0.0010_{\exp } .
\end{aligned}
$$

The $\mathcal{O}\left(p^{6}\right)$ LECs in Eq. (8) also appear in the slope of the scalar form factor $\lambda_{0}$ [17] defined by $f_{0}(t)=$ $f_{+}(0)\left(1+\lambda_{0} t / M_{\pi^{+}}^{2}+\ldots\right)$ and we obtain :

$\lambda_{0}=(13 \pm 3) \cdot 10^{-3}$,

in excellent agreement with a recent experimental measurement 18 .

\section{Acknowledgements}

This work has been supported in part by MCYT
(Spain) under grant FPA2004-00996, by Generalitat Valenciana (Grants GRUPOS03/013, GV04B594 and GV05/015) and by HPRN-CT2002-00311 (EURIDICE).

\section{REFERENCES}

1. S. Weinberg, PhysicaA 96 (1979) 327; J. Gasser and H. Leutwyler, Annals Phys. 158 (1984) 142.

2. J. Gasser and H. Leutwyler, Nucl. Phys. B 250 (1985) 465.

3. J. Bijnens, G. Colangelo and G. Ecker, JHEP 9902 (1999) 020 arXiv:hep-ph/9902437.

4. G. Ecker, J. Gasser, A. Pich and E. de Rafael, Nucl. Phys. B 321 (1989) 311.

5. G. Ecker, J. Gasser, H. Leutwyler, A. Pich and E. de Rafael, Phys. Lett. B 223 (1989) 425.

6. V. Cirigliano, G. Ecker, M. Eidemuller, A. Pich, R. Kaiser and J. Portolés, "Towards a large- $N_{C}$ estimate of the $\mathcal{O}\left(p^{6}\right)$ chiral low energy couplings", work in preparation.

7. G. 't Hooft, Nucl. Phys. B 75 (1974) 461; E. Witten, Nucl. Phys. B 160 (1979) 57.

8. M. Knecht and A. Nyffeler, Eur. Phys. J. C 21 (2001) 659 arXiv:hep-ph/0106034.

9. A. Pich, in Proceedings of the Phenomenology of Large $N_{C}$ QCD, edited by R. Lebed (World Scientific, Singapore, 2002), p. 239, arXiv:hep-ph/0205030

10. M. Knecht and E. de Rafael, Phys. Lett. B 424 (1998) 335 arXiv:hep-ph/9712457; S. Peris, M. Perrottet and E. de Rafael, JHEP 9805, 011 (1998) arXiv:hep-ph/9805442.

11. J. Bijnens, E. Gamiz, E. Lipartia and J. Prades, JHEP 0304 (2003) 055 arXiv:hep-ph/0304222.

12. P. D. Ruiz-Femenia, A. Pich and J. Portolés, JHEP $\mathbf{0 3 0 7}$ (2003) 003 arXiv:hep-ph/0306157.

13. V. Cirigliano, G. Ecker, M. Eidemuller, A. Pich and J. Portolés, Phys. Lett. B 596 (2004) 96 arXiv:hep-ph/0404004.

14. V. Cirigliano, G. Ecker, M. Eidemuller, R. Kaiser, A. Pich and J. Portolés, JHEP 0504 (2005) 006 arXiv:hep-ph/0503108.

15. J. Gasser and H. Leutwyler, Nucl. Phys. B 250 (1985) 517. 
16. H. Leutwyler and M. Roos, Z. Phys. C 25, 91 (1984).

17. J. Bijnens and P. Talavera, Nucl. Phys. B 669, 341 (2003) arXiv:hep-ph/0303103.

18. T. Alexopoulos et al. $[\mathrm{KTeV}$ Collaboration], Phys. Rev. D $\mathbf{7 0}$ (2004) 092007 arXiv:hep-ex/0406003. 